\title{
RECURRENT-PROXIMAL LINEAR DIFFERENTIAL SYSTEMS WITH ALMOST PERIODIC COEFFICIENTS
}

\author{
MAHESH G. NERURKAR
}

\begin{abstract}
We consider a system of linear differential equations, $\dot{x}=$ $A(\omega \cdot t) x$, parametrized by a point $\omega \in \mathbf{T}^{2}$, the 2-torus, where $(\omega, t) \rightarrow \omega \cdot t$ denotes an irrational rotation flow on $\mathbf{T}^{2}$. We show that if the rotation number of this flow is well approximable by rationals, then residually many equations (with respect to the $C^{k}$-topology on a certain class of matrix valued maps $A(\omega)$ on $\mathbf{T}^{2}$ ) exhibit recurrent-proximal behavior. Also the order of differentiability $k$ of the class in which this generic result holds is related to the "speed" of approximation by rationals.
\end{abstract}

1. Introduction. Let $(\Omega, \mathbf{R})$ be a $C^{k}(k \in \mathbf{N})$ real flow on a compact connected $C^{\infty}$ manifold $\Omega$, and let the $\mathbf{R}$ action be denoted by $(\omega, t) \rightarrow \omega \cdot t$, $\omega \in \Omega, t \in \mathbf{R}$. Given a continuous function $A: \Omega \rightarrow M(n, \mathbf{R})$, the set of $n \times n$ real matrices, we consider a family of linear differential equations given by

$$
\dot{x}=A(\omega \cdot t) x, \quad x \in \mathbf{R}^{n} .
$$

Let $X_{A}(\omega, t): \Omega \times \mathbf{R} \rightarrow \mathrm{GL}(n, \mathbf{R})$ be the fundamental matrix solution of (1) satisfying $X_{A}(\omega, 0)=I, \forall \omega \in \Omega$. Then $X_{A}$ is a "cocycle." (A cocycle on $(\Omega, \mathbf{R})$ is a continuous map $\alpha: \Omega \times \mathbf{R} \rightarrow \operatorname{GL}(n, \mathbf{R})$ satisfying $\alpha\left(\omega, t_{1}+t_{2}\right)=\alpha\left(\omega \cdot t_{1}, t_{2}\right) \alpha\left(\omega, t_{1}\right)$ $\forall \omega \in \Omega, t_{1}, t_{2} \in \mathbf{R}$. Hence it generates a skew-product flow on $\Sigma=\mathbf{P} \times \Omega$ by setting $([x], \omega) \cdot t=\left(\left[X_{A}(\omega, t) x\right], \omega \cdot t\right)$, where $([x], \omega) \in \mathbf{P} \times \Omega$. (Here $\mathbf{P}$ is the real projective $n-1$ space and $[x]$ denotes the ray containing $\left.\left.x \in \mathbf{R}^{n}.\right)\right)$ We call system (1) recurrent-proximal if this skew-product flow on $\Sigma$ is recurrent and is a proximal extension of the flow $(\Omega, \mathbf{R})$. For easy reference we recall that a flow $(\Sigma, \mathbf{R})$ is called recurrent if the orbit of every point is dense, and the projection $\pi: \Sigma \rightarrow$ $\Omega[\pi([x], \omega)=\omega]$ is called a proximal extension if given $\left(\left[x_{1}\right], \omega_{0}\right),\left(\left[x_{2}\right], \omega_{0}\right) \in \Sigma$, there is a sequence $t_{n} \in \mathbf{R}$ such that $d\left(\left(\left[x_{1}\right], \omega_{0}\right) \cdot t_{n},\left(\left[x_{2}\right], \omega_{0}\right) \cdot t_{n}\right) \rightarrow 0$ as $n \rightarrow \infty$ (here $d$ is a metric that generates the natural product topology on $\Sigma$ ). Qualitatively this means that for any $\omega \in \Omega$ the angle between any two solutions of (1) tend to 0 for some sequence $t_{n}$ of time.

If $A(\omega) \equiv A$ is a constant matrix, then system (1) cannot be recurrent and proximal. This is because recurrence requires eigenvalues of $A$ to be purely imaginary (real eigenvectors will give rise to fixed points for the skew-product flow) but then in this case the flow on $\Sigma$ preserves distance and hence cannot be proximal. This shows (using the Floquet theorem) that if the flow on $\Omega$ is periodic, system (1) cannot be recurrent and proximal.

Received by the editors February 10, 1986. This paper was presented at the International Conference on Qualitative Properties of Differential Equations at Edmonton (June 1984).

1980 Mathematics Subject Classification (1985 Revision). Primary 58F27, 54H20; Secondary $34 \mathrm{C} 28$.

Research partially supported by N.S.F. grant DMS8401986. 
Using a technique based on developing a "smooth" version of a result of Glasner and Weiss [2], we will show that when the flow $(\Omega, \mathbf{R})$ admits "fast periodic approximations" recurrent-proximal behavior is generic. In general, results described in [2] do not have smooth analogs, the classical "small divisor" technique yields many counterexamples. Thus our technique and result is in some sense "opposite" of "small divisor" type results, furthermore they will demonstrate applicability of the "cocycle techniques" to the qualitative study on linear differential systems.

Linear differential equations which are not "almost reducible" usually show recurrent proximal behavior (see [4] for a concrete example). In [1] techniques of topological dynamics are used to prove the existence of such systems but their method cannot yield any generic result about proximal recurrent systems.

2. Notation and statement of the theorem. We have already defined a cocycle. A cocycle $X_{A}$ is $C^{k}$ (i.e. $k$ times continuously differentiable) if the map $\omega \rightarrow X(\omega, t)$ is $C^{k}$, for each $t \in \mathbf{R}$. Let $Z_{k}(\Omega)$ denote the set of all $C^{k}$ cocycles on $\Omega$. Note that the cocycle $X_{A}$ determined by system (1) uniquely determines $A$, too (in fact $\left.A(w \cdot t)=\left(d X_{A}(\omega, t) / d t\right) X_{A}(\omega, t)^{-1}\right)$. In this way, we will here onwards identify the set $Z_{k}(\Omega)$ with linear systems $\dot{x}=A(\omega \cdot t) x$.

Every $C^{k}$ map $L: \Omega \rightarrow \operatorname{GL}(n, \mathbf{R})$ gives rise to a cocycle $1^{L} \in Z_{k}(\Omega)$ by setting $1^{L}(\omega, t)=L(\omega \cdot t) L(\omega)^{-1}$. Cocycles of this type are called coboundaries and we will denote the set of all coboundaries by $B_{k}(\Omega)$. Cocycle $1^{1}$ generated by the map $\omega \rightarrow 1$ (the identity matrix) will be denoted by 1 . Given $X_{A} \in Z_{k}(\Omega)$ and $1^{L} \in B_{k}(\Omega)$, set $X_{A} \cdot 1^{L}(\omega, t)=L(\omega \cdot t) X_{A}(\omega, t) L(\omega)^{-1}$. It is easy to verify that $X_{A} \cdot 1^{L} \in Z_{k}(\Omega)$. Given $X_{A}, X_{B} \in Z_{k}(\Omega)$ we call them cohomologous if $X_{B}=X_{A} \cdot 1^{L}$ for some $1^{L} \in B_{k}(\Omega)$. We remark that $X_{B}$ and $X_{A}$ are cohomologous iff $\dot{x}=A(\omega \cdot t) x$ and $\dot{x}=B(\omega \cdot t) x$ are "kinematically similar."

Let $D$ denote the usual $C^{k}$ metric on $C^{k}(\Omega, \mathrm{GL}(n, \mathbf{R}))$, the space of all $C^{k}$ maps from $\Omega$ to $\operatorname{GL}(n, \mathbf{R})$. Then setting $D\left(X_{A}, X_{B}\right)=\sup _{0 \leq t \leq 1} D\left(X_{A}(\omega, t), X_{B}(\omega, t)\right)$ yields a complete separable metric on $Z_{k}(\Omega)$. Metrics on all other spaces will be denoted by the letter $d$. We are now ready to state our main theorem.

THEOREM (2.1). Let $\Omega=\mathbf{T}^{2}$, the 2-torus, and let the flow on $\Omega$ be generated by the line $t \rightarrow(\beta t, \alpha t)$, where $\alpha$ and $\beta$ are rationally independent irrationals satisfying the following condition.

There exists a sequence $\left(p_{n} / q_{n}\right)_{n \in \mathbf{N}}$ of irreducible fractions with $q_{n} \rightarrow \infty$ such that $\left|\beta / \alpha-p_{n} / q_{n}\right| \leq M /\left(q_{n}\right)^{2 k+1+\gamma} \forall n$, where $k \in \mathbf{N}$, and $M$ and $\gamma$ are positive constants.

Then the set $\left\{X_{A} \in \overline{B_{k}(\Omega)} \mid \dot{x}=A(\omega \cdot t) x\right.$ is recurrent-proximal $\}$ is residual in $\overline{B_{k}(\Omega)}$. (Here $\overline{B_{k}(\Omega)}$ denotes the closure of $B_{k}(\Omega)$ in $C^{k}$ topology on $Z_{k}(\Omega)$.)

REMARKS. (1) Note that if $X_{A} \in B(\Omega)$, then $\dot{x}=A(\omega \cdot t) x$ is kinematically similar to the trivial system $\dot{x}=0$ and hence cannot be recurrent-proximal.

(2) The above theorem can be generalized to the case when $\Omega=\mathbf{T}^{n}$, the $n$-torus, and the flow is generated by the line $t \rightarrow\left(\alpha_{1} t, \alpha_{2} t, \ldots, \alpha_{n} t\right)$, where the irrationals $\alpha_{1}, \ldots, \alpha_{n}$ satisfy similar "Liouville type" conditions. This situation corresponds to linear systems with quasi-periodic ( $n$-frequency) coefficients. We will however restrict ourselves to $n=2$ to keep the paper short. 
3. Proof of Theorem (2.1). First we introduce some notation. Given a cocycle $X_{A} \in Z_{k}(\Omega)$, define a homeomorphism $H\left[X_{A}: t\right]$ on $\Sigma$ by setting

$$
H\left[X_{A}: t\right]([x], \omega)=\left(\left[X_{A}(\omega, t) x\right], \omega \cdot t\right) \quad \text { for each } t \in \mathbf{R} .
$$

Also given a $C^{k} \operatorname{map} L: \Omega \rightarrow \operatorname{GL}(n, \mathbf{R})$, define $\hat{L}([x], \omega)=\left(\left[L^{-1}(\omega) x\right], \omega\right)$. Note that

$$
H\left[X_{A} \cdot 1^{L}: t\right]=\hat{L}^{-1} H\left[X_{A}: t\right] \hat{L} .
$$

Given an open set $U \subseteq \Sigma$, define $R(U)=\left\{X_{A} \in \overline{B_{k}(\Omega)} \mid \bigcup_{t \in \mathbf{R}} H\left[X_{A}: t\right](U)=\Sigma\right\}$. Let $\omega_{0}=\left(e^{i \pi}, e^{i \pi}\right) \in \Omega$. Given open sets $V_{1}, V_{2} \subset \mathbf{P}$ and $\delta>0$ define

$$
\begin{array}{r}
S\left(V_{1}, V_{2}, \delta\right)=\left\{X_{A} \in \overline{B_{k}(\Omega)} / \operatorname{diam}\left[H\left[X_{A}: t\right]\left(V_{1} \times\left\{\omega_{0}\right\}\right) \cup H\left[X_{A}: t\right]\left(V_{2} \times\left\{\omega_{0}\right\}\right)\right]\right. \\
<\delta \text { for some } t \in \mathbf{R}\} .
\end{array}
$$

Let $\mathcal{U}$ be a countable base for $\Sigma$. Then it is easy to verify that $X_{A} \in \bigcap_{U \in \mathcal{U}} R(U)$ implies that $\dot{x}=A(\omega \cdot t) x$ is recurrent.

Similarly if $X_{A} \in \bigcap_{U \in \mathcal{U}} \bigcap_{V \in \mathcal{U}} \bigcap_{n=1}^{\infty} S(U, V, 1 / n)$, then any pairs of points in the fiber over $\omega_{0}$ is proximal and since $(\Omega, \mathbf{R})$ is recurrent, this implies that $\dot{x}=A(\omega \cdot t) x$ is proximal.

Thus our proof will be complete (using Baire category theorem) by showing that each $R(U)$ and $S\left(V_{1}, V_{2}, \delta\right)$ is open and dense in $\overline{B_{k}(\Omega)}$. Openness is easy to verify, so we turn to the proof of their density.

PROOF OF DENSITY OF $R(U)$. From (*) it follows that $X_{A} \cdot 1^{L} \in R(U)$ iff $X_{A} \in R\left(\hat{L}^{-1}(U)\right)$. Thus if we show that $1 \in \overline{R(U)}$ for each open $U$, it follows that $1^{L} \in \overline{R(U)} \forall$ open $U$ and $\forall C^{k}$ maps $L$ from $\Omega$ to $\operatorname{GL}(n, \mathbf{R})$ and this implies $\overline{R(U)}=\overline{B_{k}(\Omega)}$ for any open $U$. Now note that to show $1 \in \overline{R(U)}$ it is enough to prove the following lemma.

LEMMA (3.1). Given an open set $U \subset \Sigma$ and $\varepsilon>0$, there exists a $C^{k}$ map $L: \Omega \rightarrow \mathrm{GL}(n, \mathbf{R})$ such that

(a) $D\left(1^{L}, 1\right)<\varepsilon$ and

(b) $\bigcup_{t \in \mathbf{R}} H\left[1^{L}: t\right](U)=\Sigma$.

Proof. Let $V \subseteq \mathbf{P}$ and $W \subseteq \Omega$ be open sets such that $V \times W \subseteq U$. Pick $G_{1}, \ldots, G_{n} \in \mathrm{GL}(n, \mathbf{R})$ such that

(1) $\bigcup_{i=1}^{m} G_{i}(V)=\mathbf{P}$, where $G_{i}(V)=\left\{\left[G_{i} x\right] /[x] \in V\right\}$.

(2) Let $h:[0,1] \rightarrow \mathrm{GL}(n, \mathbf{R})$ be a $C^{k}$ map such that $\left\{G_{1}, \ldots, G_{m}\right\} \in h[0,1]$.

Now select $N \in \mathbf{N}$ such that

(3)(i) $\tilde{B} M / q_{N}^{\gamma}<\varepsilon$, where $\tilde{B}$ is some constant (which will be described later) depending only on $k$ and the function $h$.

(ii) The open set $W$ contains a ball of radius $1 / q_{N}$ (here the radius is with respect to that metric obtained by identifying $\Omega$ with the square $[0,1] \times[0,1]$ whose opposite edges are identified).

From hereon we will identify $\Omega=\mathbf{T}^{2}$ with the square $\left[0, p_{N}\right] \times\left[0, p_{N}\right]$ whose opposite edges are identified, and addition of any real numbers will mean addition $\bmod p_{N}$. Our required map $L$ will be of the form $L=h \circ \theta$, where 


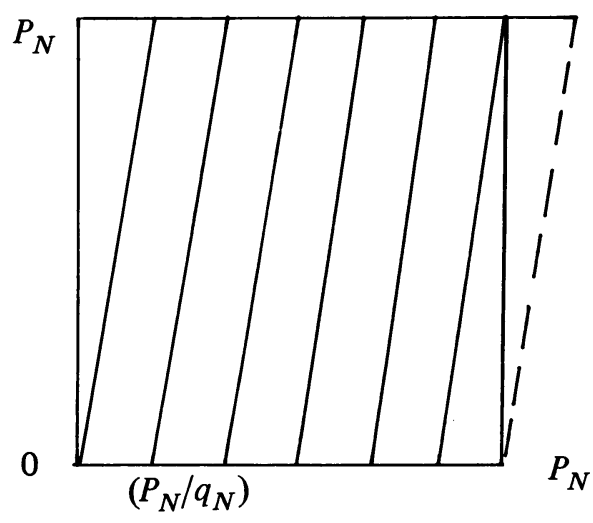

FIGURE 1

$\theta:\left[0, p_{N}\right] \times\left[0, p_{N}\right] \rightarrow[0,1]$ will be defined shortly. To construct $\theta$, first divide $\mathbf{T}^{2}$ into parallelograms as shown in Figure 1.

Let $\bar{\theta}(x)=\sin \left(2 \pi q_{N} x / p_{N}\right) \forall x \in \mathbf{R}$, and set $\theta(x, y)=\bar{\theta}\left(x-p_{N} y / q_{N}\right)$. Note that, (**)

$$
\theta\left(x+p_{N} t / q_{N}, y+t\right)=\theta(x, y) \text {. }
$$

Clearly $\theta$ is a $C^{\infty}$ map on $\mathbf{T}^{2}$. We now show that $L=h \circ \theta$ satisfies (b). Since

$$
\bigcup_{t \in \mathbf{R}} H\left[1^{L}: t\right](U)=\bigcup_{t \in \mathbf{R}} \hat{L}^{-1} H[1: t] \hat{L}(U),
$$

to prove (b) it is enough to check that $\bigcup_{t \in \mathbf{R}} H[1: t] \hat{L}(U)=\Sigma$. Now given any $[x] \in \mathbf{P}$, by (1) and the fact that $\theta(W)=[0,1]$ (by (3)(ii)), it follows that $\exists \omega \in W$ such that $([x], \omega) \in \hat{L}^{-1}(V \times W)$, that is $\hat{L}([x], \omega) \in U$. Also since $(\Omega, \mathbf{R})$ is recurrent, $\bigcup_{t \in \mathbf{R}} H[1: t]([x], W)=[x] \times \Omega$. These two observations imply that $\bigcup_{t \in \mathbf{R}} H[1: t] \hat{L}(U)=\Sigma$, proving (b).

To prove (a), we first state some general facts. (1) The $C^{i}$ norm $\|f\|_{i}$ of the lift of $f \in C^{i}\left(\mathbf{T}^{2}\right)$ to $\left[0, P_{N}\right] \times\left[0, P_{N}\right]$ is $P_{N}^{i}$ times the $C^{i}$ norm on $C^{i}\left(\mathbf{T}^{2}\right)$. (2) Given a manifold $M$, a Lie group $G$, and $C^{k}$ maps $f: \mathbf{R} \rightarrow G, f_{i}: M \rightarrow G$ and $g, g_{i}: M \rightarrow \mathbf{R}(i=1,2)$, we have

$$
D\left(f \circ g_{1}, f \circ g_{2}\right) \leq B_{1}\left\|g_{1}-g_{2}\right\|_{k}
$$

and

$$
D\left(f_{1} \cdot[f \circ g], f_{2} \cdot[f \circ g]\right) \leq B_{2} D\left(f_{1}, f_{2}\right)\|g\|_{k} .
$$

[Here $D$ is the $C^{k}$ metric, o denotes the composition, and - is the group multiplication.] Note that the constant $B_{1}$ depends on the function $f$ and the number of terms one gets in computing $k$-derivatives of composition of two functions using product and chain rule. Thus $B_{1}$ depends on $f$ and $k$. Similarly $B_{2}$ depends only on $f$ and $k$ too.

Now fix $t \in[0,1]$; applying (1) and (2)(ii) with $f_{1}(\omega)=L(\omega \cdot t), f_{2}=L(\omega)$, $f=h$, and $g=\theta$ we get

$$
\begin{aligned}
D\left(1^{L}, 1\right) & =\operatorname{Sup}_{0 \leq t \leq 1} D\left(L(\omega \cdot t) L(\omega)^{-1}, L(\omega) L(\omega)^{-1}\right) \\
& \leq P_{N}^{k} B_{2} D(L(\omega \cdot t), L(\omega))\|\theta\|_{k}
\end{aligned}
$$


Then applying (1) and (2)(i) with $f=h, g_{1}=\theta(\cdot t)$, and $g_{2}=\theta$ we get

$$
D\left(1^{L}, 1\right) \leq P_{N}^{2 k} B \operatorname{Sup}_{0 \leq t \leq 1}\|\theta[(x, y) \cdot t]-\theta(x, y)\|_{k}\|\theta\|_{k},
$$

where $\omega=(x, y)$ and $B=B_{1} B_{2}$ is a constant depending only on $h$ and $k$.

$$
\begin{aligned}
D\left(1^{L}, 1\right) & \leq P_{N}^{2 k} B\left(2 \pi q_{N} / p_{N}\right)^{2 k+1} \cdot \alpha\left|\beta / \alpha-p_{N} / q_{N}\right|, \text { by the mean value theorem, } \\
& \leq \tilde{B} q_{N}^{2 k+1}\left|\beta / \alpha-p_{N} / q_{N}\right|, \quad \text { where } \tilde{B}=(2 \pi)^{2 k+1} B \alpha\left(\text { note that } p_{N} \geq 1\right) \\
& \leq \tilde{B} M / q_{N}^{\gamma}<\varepsilon \text { by }(3) .
\end{aligned}
$$

This completes the proof of density of $R(U)$.

Density of $S\left(V_{1}, V_{2}, \delta\right)$. As before it is enough to show that $1 \in \overline{S\left(V_{1}, V_{2}, \delta\right)}$ $\forall V_{1}, V_{2}$, and $\delta$. This means that given $\varepsilon>0$ we want to find a $C^{k}$ map $L: \Omega \rightarrow$ $\operatorname{GL}(n, \mathbf{R})$ such that (a) $D\left(1^{L}, 1\right)<\varepsilon$ and (b) $1^{L} \in S\left(V_{1}, V_{2}, \delta\right)$. As before the map $L$ will be of the form $L=h \circ \theta$, where $\theta: \mathbf{T}^{2} \rightarrow[0,1]$ is exactly the same map constructed in the previous proof and $h:[0,1] \rightarrow \operatorname{GL}(n, \mathbf{R})$ is a $C^{k}$ map such that $h(0)=h(1)=1$, the identity matrix, and $h(1 / 2)=G_{0}^{-1}$, where $G_{0} \in \operatorname{GL}(n, \mathbf{R})$ is such that $\operatorname{diam} G_{0}\left(V_{1} \cup V_{2}\right)<\delta / 2$ (such that a $G_{0}$ can be found, see [2] for details). Note that $\hat{L}\left(\omega_{0}\right)=G_{0}$, hence $\operatorname{diam}\left[\hat{L}\left(V_{1} \times\left\{\omega_{0}\right\} \cup V_{2} \times\left\{\omega_{0}\right\}\right)\right]<\delta / 2$. This implies that diam $H[1: t]\left(\hat{L}\left(V_{1} \times\left\{\omega_{0}\right\} \cup V_{2} \times\left\{\omega_{0}\right\}\right)\right)<\delta / 2$. Since $H\left[1^{L}: t\right]=L(\omega \cdot t) L(\omega)^{-1}$, pick $t \in \mathbf{R}$ such that $\omega_{0} \cdot t$ is close to $(0,0) \in \mathbf{T}^{2}$. By our choice of $h$, this implies that $L\left(\omega_{0} \cdot t\right)$ is close to the identity matrix. In fact choose $t$ so that $L\left(\omega_{0} \cdot t\right)$, as a map on $\mathbf{P}$, takes a set of diameter less that $\delta / 2$ to a set with diameter no more than $\delta$. This implies that $1^{L} \in S\left(V_{1}, V_{2}, \delta\right)$, proving the density of $S\left(V_{1}, V_{2}, \delta\right)$. This completes the proof of Theorem (2.1).

REMARK. Suppose the rotation number $\beta / \alpha$ of the flow on $\Omega$ is "badly approximable." Then we do not know whether one can have recurrent-proximal systems based on $\Omega$. In this case we conjecture that if $A(\omega)$ is smooth enough and all Lyapunov exponents of system (1) are zero, then system (1) cannot be recurrentproximal (the restriction of Lyapunov exponents being zero stems from the fact that we want our corresponding cocycle to be in the closure of coboundaries). If proved this will be a noncommutative analog of the classical Kolmogorov-Siegel Theorem.

\section{REFERENCES}

1. R. Ellis and R. Johnson, Topological dynamics and linear differential systems, J. Differential Equations 44 (1982), 21-39.

2. S. Glasner and B. Weiss, On the construction of minimal skew products, Israel J. Math. 34 (1979).

3. R. Johnson, Ergodic theory and linear differential equations, J. Differential Equations 28 (1978), 23-34.

4. V. Millionshchikov, Proof of the existence of irregular systems of linear differential equations with almost periodic coefficients, Differential Equations 4 (1968).

Department of Mathematics, Ohio State University, Columbus, Ohio 43210 